\title{
BMJ Open TeleNeurological evaluation and Support for the Emergency Department (TeleNS-ED): protocol for an open-label clinical trial
}

\author{
Jessica Mandrioli (1) , ${ }^{1,2}$ Mario Santangelo, ${ }^{3}$ Antonio Luciani, ${ }^{4}$ Stefano Toscani, ${ }^{5}$ \\ Elisabetta Zucchi, ${ }^{2}$ Giada Giovannini, ${ }^{1,2}$ Ilaria Martinelli, ${ }^{1}$ Sonia Cecoli, ${ }^{6}$ \\ Guido Bigliardi, ${ }^{1}$ Sara Scanavini, ${ }^{7}$ Stefano Meletti ${ }^{1,2}$
}

To cite: Mandrioli J,

Santangelo M, Luciani A, et al. TeleNeurological evaluation and Support for the Emergency Department (TeleNS-ED): protocol for an open-label clinical trial. BMJ Open 2021;11:e048293. doi:10.1136/ bmjopen-2020-048293

- Prepublication history for this paper is available online. To view these files, please visit the journal online (http://dx.doi org/10.1136/bmjopen-2020048293).

Received 22 December 2020 Accepted 06 May 2021

Check for updates

(c) Author(s) (or their employer(s)) 2021. Re-use permitted under CC BY-NC. No commercial re-use. See rights and permissions. Published by BMJ.

For numbered affiliations see end of article.

Correspondence to Professor Stefano Meletti; stefano.meletti@unimore.it

\section{ABSTRACT}

Introduction The COVID-19 pandemic compelled health systems to protect patients and medical personnel during transit in hospitals by minimising transfers, prompting the use of telehealth systems. In the field of neurology, telemedicine has been used in emergency settings for acute stroke management between spoke and hub hospital networks, where good outcomes have been achieved. However, data on the use of telemedicine in non-stroke acute neurological conditions accessing the emergency department (ED) are currently missing. Methods and analyses This is an interventional, openlabel trial on the use of teleconsultation in the ED for neurological diseases other than stroke. The study aims to develop a remote consultancy system (TeleNeurological Evaluation and Support, TeleNS) for patients with acute neurological symptoms referred to hospital facilities without a 24-hour availability of a neurologist consultant (spoke hospitals). The study population will include 100 ED patients referred to two spoke hospitals in 6 months, who will be asked to perform teleconsultation instead of inperson visits. As a control group, retrospectively available data from patients admitted to the ED of spoke hospitals during the same time period over the last 2 years will be evaluated. The primary objective is to assess whether a TeleNS for the ED guarantees a faster but qualitatively non-inferior diagnostic/therapeutic work-up if compared with inperson examination, assuring the availability of all the necessary examinations and treatments with consistent time-saving.

Ethics and dissemination The trial was designed following the national guidelines on clinical investigation on telemedicine provided by the Italian Ministry of Health and according to the Standard Protocol Items for Randomized Trials statement guidelines. This research protocol was approved by Comitato Etico Area Vasta Emilia Nord in September 2020 (number/identification: 942/2020/DISP/AOUMO SIRER ID 805) and was written without patient involvement. Patients' associations will be involved in the dissemination of study design and results. The results of the study will be presented during scientific symposia or published in scientific journals.

Trial registration number NCT04611295.
Strengths and limitations of this study

- Our protocol assesses the clinical feasibility of TeleNeurological Evaluation and Support for nonstroke diseases in spoke hospitals.

- Only a few studies have assessed teleconsultation for non-stroke urgent neurological evaluation.

- This organisational model could help to further develop telemedicine and new technologies, with the COVID-19 pandemic being an unexpected opportunity to speed up the process.

- Our project has the potential to improve the quality of care and save time, while guaranteeing patient safety in the emergency department.

- This is a pilot study with a small sample size and therefore future studies should evaluate teleneurology compared with inperson visits in a larger cohort and in different settings.

\section{INTRODUCTION}

\section{Background and rationale}

Although 'telemedicine' lacks a definitive definition, the WHO has described it as:

The delivery of health care services, where distance is a critical factor, by all health care professionals using information and communication technologies (ICT) for the exchange of valid information for diagnosis, treatment and prevention of disease and injuries, research and evaluation, and for the continuing education of health care providers, all in the interests of advancing the health of individuals and their communities. ${ }^{1}$

Being an open and constantly evolving science that incorporates new advancements in technology with the aim to respond and adapt to the changing health needs and contexts of societies, telemedicine is characterised by four fundamental goals: (1) to 
provide clinical support; (2) to overcome geographical barriers, connecting users who are not in the same physical location; (3) to use various types of ICT; and (4) to improve health outcomes.

Telemedicine applications can be classified according to the timing of the information provided. For example, in asynchronous telemedicine the exchange of prerecorded data between two or more individuals occurs at different times, whereas in synchronous telemedicine individuals are simultaneously present, as in the case of teleconsulting. Another way to differentiate telehealth is considering the type of interaction between individuals, be it health professional to health professional, or health professional to patient. $^{2}$

Teleconsultation is defined as synchronous or asynchronous consultation using ICT to overcome geographical and functional distance, for diagnostics or treatment between two or more geographically separated health providers or between health providers and patients.

Many types of teleconsultation may exist, where both sides are health providers (eg, who may need a second expert opinion), both in ordinary medical setting ${ }^{3-5}$ and emergency department (ED) ${ }^{6}$

If teleconsultation is established correctly, good outcomes can be obtained: timely access to correct medical information, quality improvement in the diagnosis and treatment process, increased physician trust, and significant improvement in overall quality of healthcare. ${ }^{7}$ In the neurological field, teleconsultation is increasingly used for emergency care of patients who had a stroke between hospitals with and without specialised stroke units. ${ }^{8-12}$ However, one disadvantage of this concept is the very specific focus on one medical condition.

Other teleconsultation systems in emergency medicine department have been applied mainly to cardiology field (eg, acute myocardial infarction). ${ }^{13}$

Despite these advances, there are limited data on the adequacy and feasibility of telemedicine in other neurological urgent conditions. ${ }^{14-17}$ Only one trial has investigated the potential of telemedicine for general neurology outpatient referrals, ${ }^{18}$ whereas for neurological emergencies reports are sparse and explored only in pilot projects. ${ }^{19}{ }^{20}$ In particular, studies evaluating teleneurology as a possibility of remote consultation between different hospitals are lacking (in a hub-and-spoke or network system) for patients with acute neurological symptoms outside of the 'telestroke' context.

With this trial we are going to study the performance of a neurological teleconsultation system as a complementary structural element to the ED activity.

\section{Preliminary data}

The reorganisation of health centres in order to deliver services in an effective way by taking into account economic sustainability is a topic of increasing importance for regional and national health services in Italy. EDs are a crucial access point to hospital network facilities and their management is a critical factor in order to improve system effectiveness and efficiency.

In Italy the role of EDs is even more important than in other European countries because, in addition to real emergency and urgent services, they have to face a set of demands that instead should be managed by general practitioners. This is due to historical reasons and to the absence of appropriate fast-track programmes aimed at responding to the increasing and complex needs of the entire population, especially in view of advanced ageing.

The 2019 operational programmes of the EmiliaRomagna region require the activation of new clinical care pathways for reducing waiting times for health visits, tests and examination. ${ }^{21}$

In the ED one of the reasons for the long waiting times is the need for specialist consultation in complex cases. The delays may be even more prolonged in local hospitals (not university hospitals), where specialists may not be available at all or only at predefined times.

This is the case for patients presenting with neurological symptoms that are not life-threatening but require an urgent specialist consultation. In these cases, peripheral hospitals (spoke hospitals) without a 24-hour availability of a neurology consultant may activate transportation to a centre where a neurologist is available (hub hospital), or even admit patients in shortstay observation units where they are monitored until neurological consultation, together with other visits, is performed.

At this time two neurology units in the province of Modena offer neurological consultation for the ED in hub hospitals 24 hours a day, 7 days a week. Furthermore a consultant is also present at spoke hospitals for consultation 6 hours a day (08:30-14:30), 5 days a week (MondayFriday). Neurological consultations at spoke hospitals are not performed during night hours, on holidays and in the afternoon hours after 14:00. During these time slots, patients admitted to the spoke hospitals of the province have to be referred to neurology units for an 'in person' specialist consultation.

This requires a generally long diagnostic path which includes acceptance and general evaluation at the ED of the spoke hospital, request for neurological advice at the hub hospital and interhospital transportation. After neurological evaluation at the hub hospital, the patient can be admitted to the neurology unit or return to the $\mathrm{ED}$ of the spoke hospital, where further investigations can be carried out if indicated. Finally, the patient can undergo a short-stay observation, can be admitted to the local hospital ward or be discharged home.

The negative consequences of this long diagnostic pathway include the following:

- High risk of complications such as infections (as seen during COVID-19 pandemic) and confusion among the elderly, etc, during the waiting time at the ED.

- Frequent use of interhospital transport systems, with consequent reduction of their availability to the entire ED. 
- Long waiting time to activate ambulance transport, arrival to the neurology ambulatory (often engaged with the ED of the hub hospital) and again for the transport back to the spoke hospital.

- Safety issues regarding patient management at hub hospitals, where patients wait in the aisle without supervision, with risks of, for example, fall among the elderly with confusion.

To date, in the province of Modena (a city located in Northern Italy, counting a population of 700000 inhabitants), teleconsultation in neurology has been acquired for stroke diagnosis and treatment with the so-called 'telestroke', through which an internal medicine specialist, guided by a neurologist, could treat earlier patients who had a stroke residing in the mountainous areas with intravenous recombinant tissue-plasminogen activator. First studies from Bavaria, Germany demonstrated that telethrombolysis was a feasible and safe procedure, and in a period of 10 years more than 4000 patients who had acute ischaemic stroke had been treated. ${ }^{8-10}$

This offered the opportunity for patients residing in remote areas of the province to receive prompt evaluation and fast treatment, under the supervision of a specialised stroke neurologist, avoiding long journeys along with the unpredictability of traffic jams, and consequently delays in treatment and with the possibility of decreased efficacy and occurrence of side effects. ${ }^{22}$

Additionally, avoiding patient journey from one hospital to another saves time per visit both for the physician and the expert and enabled a more economical utilisation of resources, leaving ambulances for use in other emergencies. In these times of the COVID-19 pandemic, telemedicine systems, which spare unnecessary travel between hospitals and contacts between potentially infected persons, are now more than ever advocated by specialists and international neurology societies. ${ }^{23-26}$

The purpose of the TeleNeurological Evaluation and Support for the Emergency Department (TeleNS-ED) project is to guarantee a diagnostic-therapeutic process that is not inferior to the standard 'in person' neurological assessment in terms of accuracy and access to due treatments, in significantly faster times than the current route.

\section{METHODS AND ANALYSIS}

This is an interventional, open-label trial on the use of teleconsultation in the ED for neurological conditions other than acute stroke (for which a specific protocol already exists).

The study aims at developing a remote neurological consultancy system (teleconsultation) for patients with acute neurological symptoms who are referred to hospital facilities without a 24-hour availability of a neurologist consultant. These conditions include but are not limited to suspected recent seizures, altered mental status not explained by acute intoxications or metabolic derangements, diplopia, vertigo, new-onset headache, localised subacute loss of sensation, paraesthesia or weakness, and acute infections or metabolic alterations potentially decompensating chronic neurological diseases such as myasthenia gravis, epilepsy, Alzheimer disease, Parkinson disease, etc.

\section{Study design}

The trial was designed following the national guidelines on clinical investigations for telemedicine (http://www. salute.gov.it/imgs/C_17_pubblicazioni_2129_allegato. pdf) provided by the Italian Ministry of Health and according to the Standard Protocol Items for Randomized Trials statement guidelines. The study protocol (version 1,1 July 2020) was approved by competent authorities (Comitato Etico Area Vasta Emilia Nord in September 2020; number/identification: 942/2020/DISP/AOUMO SIRER ID 805).

The trial was registered at ClinicalTrials.gov (https:// www.clinicaltrials.gov/ct2/show/NCT04611295? cond= telens\&draw $=2 \&$ rank $=1$ ).

The planned start date was on 1 December 2020 and the end date on 10 June 2021.

\section{Study procedures and setting}

The project will be set at the two hub hospitals of the province, where neurological service will be available in the afternoon, at night and on public holidays, time windows when the neurologist consultant is not present at spoke hospitals. Figure 1 depicts the procedural flow chart of the study.

Project enrolment is expected to be achieved over a period of 6 months, when the outcome and process indicators will be assessed and compared between the following groups:

- Experimental group (cases): patients who access spoke hospitals' ED in the afternoon and night-time and on holidays.

- Control group: retrospectively collected data on neurological consultations performed by neurological hub hospitals for patients in the same time slots and in the same months of the last 2 years (ie, inperson neurological consultations performed during afternoon, night-time and holidays will be the comparator group).

\section{Objectives}

The study is designed as a non-blinded, controlled study conducted at two hub-and-spoke hospitals in Modena. This study will provide more knowledge about the effects and benefits of teleconsultation in terms of diagnostic process.

The primary objective is to assess whether TeleNS for the ED can guarantee faster but qualitatively non-inferior diagnostic/therapeutic work-up if compared with inperson examinations.

As secondary objectives, we aim to determine if neurological teleconsultation in urgent but non-life-threatening neurological cases is superior in terms of (1) effect 
EXPERIMENTAL TeleNS-ED
USUAL CLINICAL PRACTICE

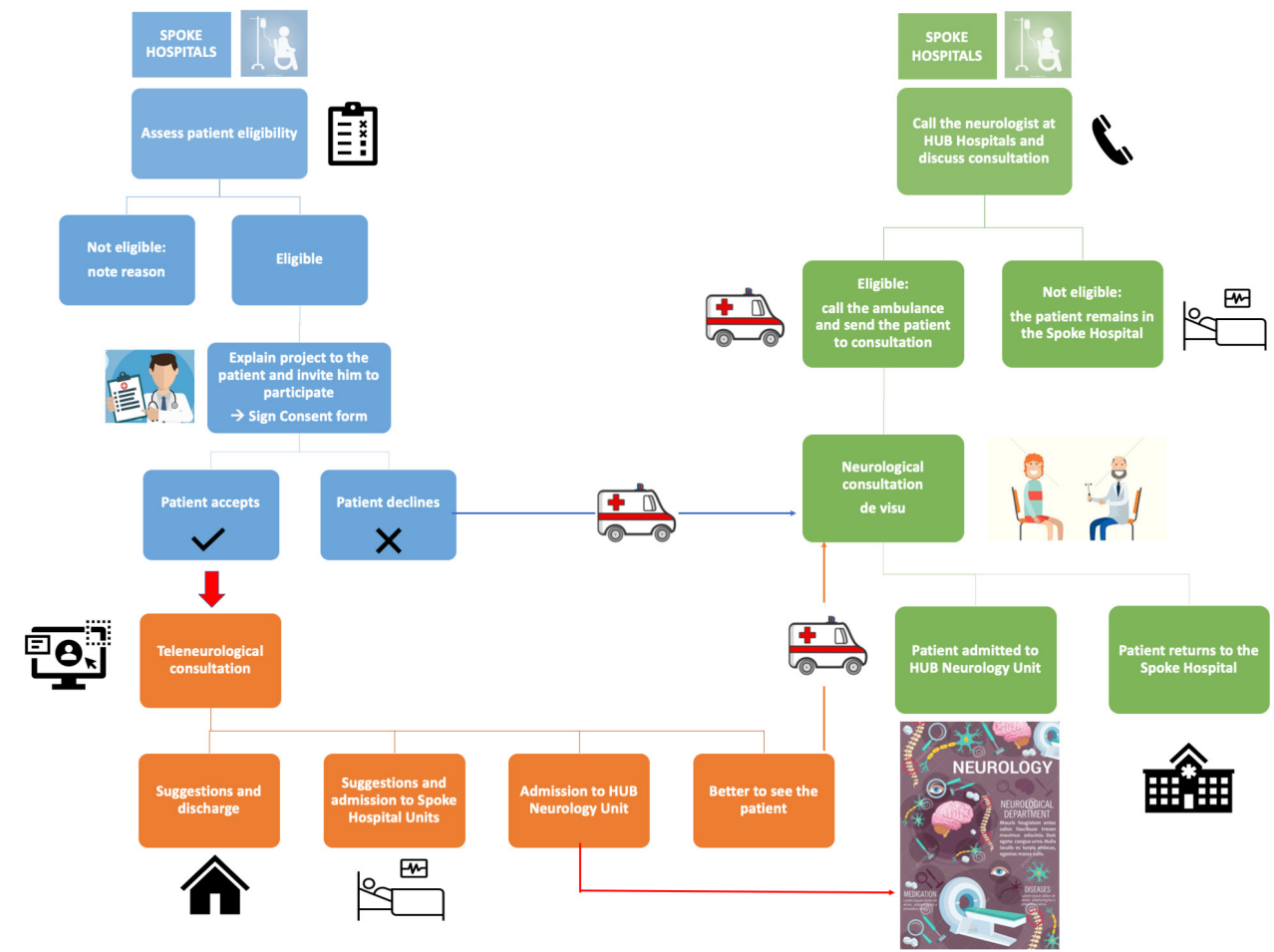

Figure 1 Design of the study. The cartoon depicts the flow chart of the study comparing experimental versus usual care treatment arms. On the left, the blue rectangles represent experimental operating procedures to be done at the emergency department before teleneurological consultation is instituted, and the orange rectangles show all the possible clinical scenarios, with the potential to switch to usual care practice. On the right, the green rectangles (usual clinical practice) demonstrate the standard care algorithm from the emergency department to inperson neurological consultation at the hub hospital with the possible outcome (admission to neurology unit/return to spoke hospital). TeleNS-ED, TeleNeurological Evaluation and Support for the Emergency Department.

evaluation, (2) process evaluation and (3) cost-benefit analysis.

\section{Primary outcome measures}

- Percentage of patients for whom teleneurological evaluation is activated over the total number of patients who access the ED during the days and time slots in which the study is active and needing neurological consultancy.

Objective: to reach at least $85 \%$ of patients.

- Percentage of patients with ED readmission in the next 7 days following the evaluation.

Objective: it is expected that for patients of the teleneurology group, the percentage of reaccess will not exceed that of patients who underwent standard neurological advice.

- Percentage of patients who will undergo hospitalisation following neurological advice.

Objective: it is expected that for patients of the teleneurology group, the percentage of hospitalisation will not exceed that of patients who underwent standard neurological advice.

- 'Door to end of diagnostic process' time, that is, time from the patient's admission to the end of the diagnostic process (total time spent in the ED).
Objective: the duration is expected to be significantly shorter for patients of the teleneurology group than for patients who underwent standard neurological advice.

- 'Door to end of neurological evaluation' time, that is, time from the patient's admission to the end of neurological consultation.

Objective: the duration is expected to be significantly shorter for patients of the teleneurology group than for patients who underwent standard neurological advice.

\section{Secondary outcome measures}

- Percentage of cases assessed by teleconsultation for which the neurologist was unable to make remote decisions and requested an 'in person' evaluation.

- Evaluation of the number and type of requests for diagnostic tests for the patients of the teleneurology group compared with the control group.

Objective: it is expected that for patients of the teleneurology group, the number of requests for intermediate services (as well as for imaging studies and inhouse services adjacent to neurology, such as neurosurgery, ophthalmology, etc) does not exceed that of patients followed with standard neurological consultancy. 
Table 1 Centres and facilities involved in the study

\begin{tabular}{|c|c|c|c|}
\hline Centre & Role and activity & PI/responsible & Work package \\
\hline $\begin{array}{l}\text { Azienda Ospedaliero } \\
\text { Universitaria dì Modena } \\
\text { (sponsor)-Neurology Unit }\end{array}$ & Neurological hub & $\begin{array}{l}\text { Professor Stefano } \\
\text { Meletti }\end{array}$ & $\begin{array}{l}\text { Coordination of the trial through monitoring of } \\
\text { the centre's activities, facilitating communication, } \\
\text { promoting exchange of ideas and } \\
\text { methodological approach, stimulating analysis } \\
\text { and integration of results. } \\
\text { Organisation of kick-off meeting and regular } \\
\text { meetings with partners. } \\
\text { Submission to ethical committees. } \\
\text { Establishment of an independent data and safety } \\
\text { monitoring board. } \\
\text { Neurological consultation by teleconsultation } \\
\text { and by inperson examination. } \\
\text { Presentation and dissemination of results. }\end{array}$ \\
\hline $\begin{array}{l}\text { Azienda Ospedaliero } \\
\text { Universitaria dì Modena } \\
\text { (Sponsor)- } \\
\text { Emergency Department }\end{array}$ & Spoke hospital & Dr Giuseppe Pezzuto & $\begin{array}{l}\text { Enrolment of } 50-60 \text { patients (ICF forms) } \\
\text { presenting to the ED due to an acute } \\
\text { neurological condition (except for stroke) fulfilling } \\
\text { the inclusion and exclusion criteria in a period of } \\
6 \text { months. } \\
\text { Presentation of the case, interface between } \\
\text { patient and neurologist, guided teleconsultation } \\
\text { and neurological examination. }\end{array}$ \\
\hline $\begin{array}{l}\text { Azienda USL dì Modena- } \\
\text { Neurology Unit }\end{array}$ & Neurological hub & Dr Mario Santangelo & $\begin{array}{l}\text { Neurological consultation by teleconsultation } \\
\text { and by inperson examination. } \\
\text { Presentation and dissemination of results. }\end{array}$ \\
\hline $\begin{array}{l}\text { Azienda USL dì Modena- } \\
\text { Emergency Department }\end{array}$ & Spoke hospital & Dr Stefano Toscani & $\begin{array}{l}\text { Enrolment of } 40-50 \text { patients (ICF forms) } \\
\text { presenting to the ED due to an acute } \\
\text { neurological condition (except for stroke) fulfilling } \\
\text { the inclusion and exclusion criteria in a period of } \\
6 \text { months. } \\
\text { Presentation of the case, interface between } \\
\text { patient and neurologist, guided teleconsultation } \\
\text { and neurological examination. }\end{array}$ \\
\hline
\end{tabular}

ED, emergency department; ICF, Informed Consent Form; PI, Principal Investigator.

- Evaluation of users' satisfaction through a Visual Analogue Scale (VAS) score for patients and caregivers.

- Evaluation of users' satisfaction through VAS score for the medical staff involved.

- Cost-benefit analysis will be performed to determine the direct costs involved in the two systems and to analyse additional data on teleconsultation, which will be collected during the study.

\section{Study population}

The study will include patients who need neurological consultation after being admitted to the ED of the spoke hospitals where a neurologist is not physically available. The study population is estimated at 100 outpatients and the enrolment period will last 6 months. These ED patients referred to spoke hospitals will be asked to perform teleconsultation instead of 'in person' neurological examination, and a written informed consent will be obtained. Retrospectively available data from patients admitted to the ED of spoke hospitals during the same daytime and months (of the experimental group) of the last 2 years will be used as the control group. Centres and facilities involved in the study are shown in table 1 .

Figure 1 shows the design of the study.

\section{Eligibility criteria}

Inclusion criteria

- Patients aged $>18$ years.

- Symptoms related to possible acute/subacute neurological pathology, or worsening of known neurological pathology, for which the ED physician considers neurological consultation indicated/necessary.

- Signed informed consent to remote neurological evaluation.

\section{Exclusion criteria}

- All life-threatening emergency cases where the study procedure would interfere with clinical practice.

- Patients with known neurological pathology or with clinical severity to such an extent that the need for admission to a hub neurology unit is immediately evident. 
- Refusal of the patient/caregiver to perform remote neurological evaluation.

\section{Sample size calculation}

As a pilot study we aim to enrol 100 participants in the trial, and the participating centres have committed to recruit patients according to this goal. The sample size was decided based on estimates of neurological consultations required in our local healthcare system. Power calculations were not used to calculate how many patients should be included in the trial.

Our internal reports count that at least one patient per day is referred to each neurological unit set at the two hub hospitals. In 2019, $750 \mathrm{ED}$ patients in the spoke hospitals were evaluated by a neurologist. Of these, 300 were evaluated not at the spoke hospitals but at the hub hospitals because these were outside the time slots during which the neurologist was present.

Considering the possible refusal of the system by the patients and the possible initial difficulties of the personnel in using the system, we think that a sample size of 100 will be achieved in 6 months and will be enough to estimate significant effects of teleconsultation on health, process and costs.

Should patient enrolment be faster than expected, additional patients will be recruited until the end of the 6-month study. In the case of a slower patient enrolment, we will consider extending the study period.

\section{Study intervention and teleconsultation system}

The technology employed for this study (InTouch Lite Remote Presence System) is provided by InTouch Health (Santa Barbara, California; www.intouchhealth.com). An InTouch system comprised Provider Access and Patient Access (InTouch devices).

The two devices are linked via the internet over the secure InTouch Telehealth Network. With Provider Access devices, the physician can access designated patient devices by two-way audio and video communications, allowing the best of remote interaction. The InTouch Lite is a Food and Drug Administration class II A cleared device.

\section{Teleconsultation training}

All ED and neurological unit physicians will be instructed by standard operative procedures prior to patients' enrolment. To allow for an effective telemedicine consultation, the involved physicians should feel comfortable with the concepts of telemedicine and the equipment, as well as with the videoconferencing system. A specific training on the InTouch Lite medical device will be performed in order to guarantee successful and effective examination.

\section{Equipment security}

InTouch Lite will be stored and used in specifically identified locations inside the ED (the teleconsultation box) where it can remain safe and secure. ED telemedicine coordinators and remote telemedicine presenters are responsible for the general security of the equipment at their location. The equipment will only be used for this study purpose. When not in use, it will be secured by the presence of healthcare professionals in the ED.

For specific technical equipment malfunctions, a clinical engineering service is available and insurance coverage has been prepared.

\section{Confidentiality}

The same standards, procedures and privacy practices of an inperson examination of a patient will be applied to teleconsultation, in line with Italian national policies and laws.

The viewing of a live consultation by any third party not directly connected with the consultation will need to be approved by the study coordinator. This does not apply to technical and operational staff or persons necessary to facilitate the consultation, nor students and residents who would view face-to-face patient examinations in the course of their education. Such viewing by students should be conducted in accordance with the usual and customary policies and practices of students viewing or participating in face-to-face examinations. The same standards of professionalism and medical care apply to telemedicine examinations.

\section{Data management and analysis}

Data will be collected and linked from several sources at several points in time. A data management plan will be developed and shared with the scientific board.

Data will be collected in a dedicated database, which will be locked at the end of the study.

$\chi^{2}$ test will be used to explore differences between groups for categorical data and t-test (or multiple comparison test) for continuous data. Correlations will be examined using Pearson coefficient. For some variables we will use non-parametric tests such as Wilcoxon signed-rank test or McNemar test. In these tests, each of the collected variables is examined separately, and the null hypothesis is that there is no difference between the intervention and the control group. We will use the conventional significance level of $5 \%$ in the statistical analyses.

For some (groups of) variables, it will be relevant to use different types of regression models to study the effect of teleconsultation. Both difference-in-difference models and structural equation models will be applied. The use of structural equation models will allow the study of how teleconsultation affects a group of variables simultaneously.

\section{Strength and risk analysis}

This is the first interventional trial assessing the efficacy of telemedicine for acute, non-vascular, neurological conditions in the setting of a spoke-and-hub hospital network. As the SARS-CoV-2 pandemic has strained local and national healthcare systems to find new solutions to minimise patients' risk for infection, as well as to protect medical and paramedical personnel, telemedicine has emerged as a key answer to the current situation. While in Italy concerns around insurance reimbursement are 
not an issue due to the national health system coverage, a possible limitation to telehealth widespread utilisation could be the access and confidence to technological devices. ${ }^{27}$ However, this emergency had profound repercussions on the care of acute medical conditions, as earlier population studies reported a dramatic delay in search for medical advice and access to ED due to patients' and carers' fear of contagion, even in the case of the most life-threatening medical conditions. ${ }^{28-30}$ As it is mandatory for health institutions to address such general population concerns, warranting the delivery of due care under the safest conditions, telemedicine suddenly regained public attention, pushing search on technological devices as well. ${ }^{25}{ }^{31}$ Although our trial did not involve patients on the other side of the remote communication devices, we expect this project to favour the spread of technological utilisation among healthcare workers and foster confidence in telehealth both for physicians and patients.

A potential drawback of the study is the exclusion of acute life-threatening non-stroke emergencies. The rationale for not including these conditions (such as status epilepticus) in the trial is partly inherent to our specific local setting. For these conditions, with an out-of-hospital onset, the patient is in fact referred directly to the hub hospital, which is available 24 hours . However, should the interhospital teleconsultation system prove to be effective, it will be important to extend teleconsultation to additional medical conditions.

A potential criticism to the trial design is that patients (or their carers) may perceive, in the setting of an emergency visit, the assignment of a teleconsultation instead of an inperson visit as a subordination of their problems and be dissatisfied with the care received. However, we trained our participating physicians to properly explain that patients are free to retrieve consent and follow the usual care route without consequences. Another potential risk is a possible selection bias towards patients who are more likely to accept telehealth under the emergent pandemic situation and more inclined to use technological devices, which may influence the cost-benefit analysis. Nonetheless, given the pivotal nature of our trial we will take into consideration the possibility of this bias, and after releasing our results to the general public we will include patients' associations in the critical evaluation of telemedicine utilisation by health systems in order to improve and advance the dissemination of this means, even for the most elderly.

We recognise teleconsultation acceptance and diffusion are also dependent on the accessibility of the local hospital networks, and our results may not be generalised to other national or international realities. Nevertheless, the nature of this project is pivotal, and we hope to foster further search on teleconsultation with future trials that include larger cohorts from different hospital networks and with longer duration of enrolment in order to observe the general public attitude towards ED during periods of COVID-19 containment.
As far as recruitment of participants is concerned, there is worry that the recruitment target will not be reached within June 2021. After 2 and 4 months from the start of the study, we will evaluate the recruitment process. If recruitment is insufficient, one option may be to involve other EDs in the province of Modena, extend the trial to 9 months or alternatively terminate the trial.

Finally, this teleconsultation study is less rigorously designed than many conventional studies. There is variation in the delivery of the intervention (in terms of medical staff) in the control group and in the study population, which may generate heterogeneity in the trial. This poses some challenges to data analysis, and the effects must be interpreted as averages over different types of interventions and groups of patients.

\section{Patient and public involvement}

This research protocol was written without patient involvement. Patients were not invited to comment on the study design and were not consulted to develop patientrelevant outcomes or interpret the results. Patients were not expected to contribute to conducting this study and to the writing of this document for readability or accuracy. Patients' associations will be involved in the dissemination of study design at start of patient enrolment to allow patient participation. Patients' associations will also be involved in the dissemination of study results not only to participants but to the entire patient communities (eg, by website information).

\section{ETHICS AND DISSEMINATION}

The study will be carried out in accordance with the Declaration of Helsinki, as amended by the 64th World Medical Association General Assembly, Fortaleza, Brazil in October 2013.

All subjects will sign and personally date an approved informed consent form after receiving detailed written and verbal information about the reason, nature, required procedures, intended duration, and possible risks and benefits and any discomfort associated with the study. Study procedures will not be performed until written informed consent has been provided. This study will be conducted in accordance with the Declaration of Helsinki and the International Conference on Harmonisation E6 guideline (Good Clinical Practice). Both the patient information sheet and the informed consent form have been approved by the ethics committee along with the study protocol. The results of the study will be presented during scientific symposia or published in scientific journals only after review and written approval by the involved parties in full respect of the privacy of the participating subjects.

An insurance company will provide insurance coverage in case damages occur from the trial. 
Author affiliations

${ }^{1}$ Neurology Unit, Azienda Ospedaliero-Universitaria di Modena Ospedale Civile di Baggiovara, Modena, Italy

${ }^{2}$ Department of Biomedical, Metabolic and Neural Sciences, University of Modena and Reggio Emilia, Modena, Italy

${ }^{3}$ Neurology Unit, Carpi Hospital, Azienda USL di Modena, Modena, Italy ${ }^{4}$ Emergency Department, Azienda Ospedaliero Universitaria di Modena, Modena, Italy

${ }^{5}$ Emergency Department, Azienda USL Modena, Modena, Italy

${ }^{6}$ Clinical Engineering, Azienda Ospedaliero Universitaria di Modena and Azienda USL Modena, Modena, Italy

${ }^{7}$ Medical Direction, Azienda Ospedaliero Universitaria di Modena, Modena, Italy

Acknowledgements The authors thank the TeleNS-ED investigators group: Department of Neuroscience, Civil Hospital, Azienda Ospedaliero Universitaria di Modena, Modena, Italy: F Antonelli, A Ariatti, G Borzi', A Chiari, L Ciolli, ML Dell'Acqua, D Ferraro, N Fini, M Mazzoli, R Pentore, L Picchetto, R Ricceri, V Rispoli, F Rosafio, P Sola, L Vandelli, AE Vaudano, G Vinceti, F Vitetta, G Zamboni; Neurology Unit, Carpi Hospital, Azienda USL Modena, Modena, Italy: S Amidei, M Costa, L Vaghi, AM Simone, G Monti, S De Pasqua, M Devetak, E Fileccia; Emergency Department, Azienda Ospedaliero Universitaria di Modena, Modena, Italy: F Barsotti, F Brittanico, V Casolari, M D’Arienzo, E De Marte, P D'Inca', S Falcone, G Ganzerla, M Gaspari Pellei, M Lettini, S Menetti, M Morelli, S Morselli, G Moscara, M Nuzzetti, G Paltrinieri, GS Pezzuto, G Quattromini, F Renzo, A Ricciardolo, F Savorani, R Sganzerla, G Vinci, M Volpe; Emergency Department, Mirandola Hospital, Azienda USL Modena, Mirandola, Modena, Italy: M Amato, L Catino, G Davanteri, F De Luca, R Fattori, D Maccari, Y Mehd, S Menghini, D Mariani, V Mantovani, M Mortello, F Pedrabissi, I Sergi.

Contributors Conceptualisation: JM, SM. Data curation: JM, MS, AL, ST, EZ, GG, IM, SC, GB, SM. Funding acquisition: SC, SM. Investigation: JM, MS, AL, ST, EZ, GG, IM, GB, SM. Methodology: JM, MS, AL, ST, SM. Project administration: JM, MS, SS, SM. Resources: SC, SS, SM. Software: SC. Supervision: JM, MS, GB, ST, SM. Validation: JM, SM. Writing-original draft: JM, EZ, IM, SM. Writing-review and editing: JM, MS, AL, ST, SM.

Funding The authors have not declared a specific grant for this research from any funding agency in the public, commercial or not-for-profit sectors.

Competing interests None declared.

Patient and public involvement Patients and/or the public were involved in the design, or conduct, or reporting, or dissemination plans of this research. Refer to the Methods and analysis section for further details.

Patient consent for publication Not required.

Provenance and peer review Not commissioned; externally peer reviewed.

Open access This is an open access article distributed in accordance with the Creative Commons Attribution Non Commercial (CC BY-NC 4.0) license, which permits others to distribute, remix, adapt, build upon this work non-commercially, and license their derivative works on different terms, provided the original work is properly cited, appropriate credit is given, any changes made indicated, and the use is non-commercial. See: http://creativecommons.org/licenses/by-nc/4.0/.

ORCID iD

Jessica Mandrioli http://orcid.org/0000-0002-9244-9782

\section{REFERENCES}

1 World Health Organization. WHO Group Consultation on Health Telematics (1997: Geneva, Switzerland) (1998). A health telematics policy in support of WHO's Health-for-all strategy for global health development : report of the WHO Group Consultation on Health Telematics, 11-16 December. Geneva, 1997. https://apps.who.int/ iris/handle/10665/63857

2 Bove AA, Homko CJ, Santamore WP, et al. Managing hypertension in urban underserved subjects using telemedicine--a clinical trial. Am Heart J 2013;165:615-21.

3 Hersh W, Helfand M, Wallace J, et al. A systematic review of the efficacy of telemedicine for making diagnostic and management decisions. J Telemed Telecare 2002;8:197-209.

4 Hersh WR, Hickam DH, Severance SM, et al. Diagnosis, access and outcomes: update of a systematic review of telemedicine services. $J$ Telemed Telecare 2006;12 Suppl 2:3-31.
5 Deldar K, Bahaadinbeigy K, Tara SM. Teleconsultation and clinical decision making: a systematic review. Acta Inform Med 2016;24:286-92

6 Brebner JA, Brebner EM, Ruddick-Bracken H. Accident and emergency teleconsultation for primary care--a systematic review of technical feasibility, clinical effectiveness, cost effectiveness and level of local management. J Telemed Telecare 2006;12 Suppl 1:5-8.

7 Nerlich M, Balas EA, Schall T, et al. Teleconsultation practice guidelines: report from G8 global health applications Subproject 4. Telemed J E Health 2002;8:411-8.

8 Audebert HJ, Kukla C, Clarmann von Claranau S, et al. Telemedicine for safe and extended use of thrombolysis in stroke: the Telemedic pilot project for integrative stroke care (TEMPiS) in Bavaria. Stroke 2005;36:287-91.

9 Audebert HJ, Schenkel J, Heuschmann PU, et al. Effects of the implementation of a telemedical stroke network: the Telemedic pilot project for integrative stroke care (TEMPiS) in Bavaria, Germany. Lancet Neurol 2006;5:742-8.

10 Audebert HJ, Schultes K, Tietz V, et al. Long-Term effects of specialized stroke care with telemedicine support in community hospitals on behalf of the telemedical project for integrative stroke care (TEMPiS). Stroke 2009;40:902-8.

11 Demaerschalk BM, Bobrow BJ, Raman R, et al. Stroke team remote evaluation using a digital observation camera in Arizona: the initial Mayo clinic experience trial. Stroke 2010;41:1251-8.

12 Meyer BC, Raman R, Hemmen T, et al. Efficacy of site-independent telemedicine in the stroke Doc trial: a randomised, blinded, prospective study. Lancet Neurol 2008;7:787-95.

13 Sejersten M, Sillesen M, Hansen PR, et al. Effect on treatment delay of prehospital teletransmission of 12-lead electrocardiogram to a cardiologist for immediate triage and direct referral of patients with ST-segment elevation acute myocardial infarction to primary percutaneous coronary intervention. Am J Cardiol 2008;101:941-6.

14 Tso JV, Farinpour R, Chui HC, et al. A multidisciplinary model of dementia care in an underserved retirement community, made possible by telemedicine. Front Neurol 2016;7:225.

15 Rasmusson KA, Hartshorn JC. A comparison of epilepsy patients in a traditional ambulatory clinic and a telemedicine clinic. Epilepsia 2005;46:767-70.

16 Müller KI, Alstadhaug KB, Bekkelund SI. A randomized trial of telemedicine efficacy and safety for nonacute headaches. Neurology 2017;89:153-62.

17 Dorsey ER, Deuel LM, Voss TS, et al. Increasing access to specialty care: a pilot, randomized controlled trial of telemedicine for Parkinson's disease. Mov Disord 2010;25:1652-9.

18 Chua R, Craig J, Wootton R, et al. Randomised controlled trial of telemedicine for new neurological outpatient referrals. J Neurol Neurosurg Psychiatry 2001;71:63-6.

19 Skorning M, Bergrath S, Rörtgen D, et al. [E-health in emergency medicine - the research project Med-on-@ix]. Anaesthesist 2009;58:285-92.

20 Ziegler V, Rashid A, Müller-Gorchs M, et al. [Mobile computing systems in preclinical care of stroke. Results of the Stroke Angel initiative within the BMBF project PerCoMed]. Anaesthesist 2008;57:677-85.

21 Bollettino Ufficiale della Regione Emilia-Romagna (BURERT), n.241 del 24.07.2019 periodico (Parte Seconda). Regione EmiliaRomagna Piano di miglioramento dell'accesso in EmergenzaUrgenza Sanitaria - Approvazione di Linee di indirizzo alle Aziende Sanitarie. Available: https://bur.regione.emilia-romagna.it/dettaglioinserzione? $\mathrm{i}=7 \mathrm{~b} 644492 \mathrm{dfe} 2471787 f 041 \mathrm{~d} 37 \mathrm{a} 19 \mathrm{f} 2 \mathrm{e} 8$ [Accessed 9th Dec 2020].

22 Nardetto L, Dario C, Tonello S, et al. A one-to-one TeleStroke network: the first Italian study of a web-based telemedicine system for thrombolysis delivery and patient monitoring. Neurol Sci 2016;37:725-30.

23 Aan recommendations for expanded coverage of telemedicine services, 2020. Available: https://www.aan.com/siteassets/homepage/tools-and-resources/practicing-neurologist-administrators/ practice-top-5/aan-comments-to-cms-telemed-coverage.pdf [Accessed 9th Dec 2020].

24 Cohen BH, Busis NA, Ciccarelli L. Coding in the world of COVID-19: Non-Face-to-Face evaluation and management care. Continuum 2020;26:785-98.

25 George BP, Kelly AG. Rethinking regional neurologic care in the coronavirus disease 2019 era. JAMA Neurol 2020;77:1061-2.

26 von Oertzen TJ, Macerollo A, Leone MA, et al. Ean consensus statement for management of patients with neurological diseases during the COVID-19 pandemic. Eur J Neurol 2021;28:7-14.

27 Dorsey ER, Topol EJ. State of telehealth. N Engl J Med 2016;375:154-61. 
28 Czeisler Mark É, Marynak K, Clarke KEN, et al. Delay or Avoidance of Medical Care Because of COVID-19-Related Concerns - United States, June 2020. MMWR Morb Mortal Wkly Rep 2020;69:1250-7.

29 Lange SJ, Ritchey MD, Goodman AB, et al. Potential Indirect Effects of the COVID-19 Pandemic on Use of Emergency Departments for Acute Life-Threatening Conditions - United
States, January-May 2020. MMWR Morb Mortal Wkly Rep 2020;69:795-800.

30 Teo K-C, Leung WCY, Wong Y-K, et al. Delays in stroke onset to hospital arrival time during COVID-19. Stroke 2020;51:2228-31.

31 Keesara S, Jonas A, Schulman K. Covid-19 and health care's digital revolution. N Engl J Med 2020;382:e82. 